UDC: $517.518 .28: 519.642$

45 (LXXI) No.1 (2021), 49-69

DOI: $10.37560 /$ matbil21451049k

\title{
ON GENERALIZED FRACTIONAL INTEGRAL INEQUALITIES FOR FUNCTIONS OF BOUNDED VARIATION WITH TWO VARIABLES
}

\author{
ARTION KASHURI ${ }^{1}$, HÜSEYIN BUDAK ${ }^{2}$, ROZANA LIKO ${ }^{1}$, MUHAMMAD AAMIR ALI $^{3}$, \\ AND KUBILAY ÖZÇELIK ${ }^{2}$
}

\begin{abstract}
In this paper, authors establish some identities for generalized fractional integrals. Utilizing these identities, some Ostrowski and Midpoint type inequalities for generalized fractional integrals for functions of bounded variation with two variables are obtained. Moreover, some new inequalities involving $k$-Riemann-Liouville fractional integrals are presented as special cases of our main results.
\end{abstract}

\section{INTRODUCTION AND PRELIMINARIES}

The study of various types of integral inequalities has been the focus of great attention for well over a century by a number of mathematicians, interested both in pure and applied mathematics. One of the many fundamental mathematical discoveries of A. M. Ostrowski [26] is the following classical integral inequality associated with the differentiable mappings:

Theorem 1. Let $\sigma:[a, b] \rightarrow \Re$ be a differentiable mapping on $(a, b)$ whose derivative $\sigma^{\prime}:(a, b) \rightarrow \Re$ is bounded on $(a, b)$, i.e. $\left\|\sigma^{\prime}\right\|_{\infty}:=\sup _{t \in(a, b)}\left|\sigma^{\prime}(t)\right|<\infty$. Then we have the following inequality:

$$
\left|\sigma(x)-\frac{1}{b-a} \int_{a}^{b} \sigma(t) d t\right| \leq\left[\frac{1}{4}+\frac{\left(x-\frac{a+b}{2}\right)^{2}}{(b-a)^{2}}\right](b-a)\left\|\sigma^{\prime}\right\|_{\infty},
$$

for all $x \in[a, b]$.

Ostrowski inequality has applications in quadrature, probability and optimization theory, stochastic, statistics, information and integral operator theory.

2010 Mathematics Subject Classification. Primary: 26D07, 26D10, 26D15, 26B15, 26B25.

Key words and phrases. Function of bounded variation, Ostrowski inequality, generalized fractional integral. 
We recall some basic definitions as follows:

Definition 1.1. Let $\sigma \in \mathcal{L}_{1}([a, b])$. The Riemann-Liouville fractional integrals $\mathcal{J}_{a+}^{\alpha} \sigma$ and $\mathcal{J}_{b-}^{\alpha} \sigma$ of order $\alpha>0$ with $a \geq 0$ are defined by

$$
\mathcal{J}_{a+}^{\alpha} \sigma(x)=\frac{1}{\Gamma(\alpha)} \int_{a}^{x}(x-t)^{\alpha-1} \sigma(t) d t, \quad x>a
$$

and

$$
\mathcal{J}_{b-}^{\alpha} \sigma(x)=\frac{1}{\Gamma(\alpha)} \int_{x}^{b}(t-x)^{\alpha-1} \sigma(t) d t, \quad x<b,
$$

respectively. Here, $\Gamma(\alpha)$ is the Gamma function and $J_{a+}^{0} \sigma(x)=J_{b-}^{0} \sigma(x)=\sigma(x)$.

Definition 1.2. [22] Let $\sigma \in \mathcal{L}_{1}([a, b])$. The $k$-Riemann-Liouville fractional integral $\mathcal{I}_{a+, k}^{\alpha} \sigma$, and $\mathcal{I}_{b-, k}^{\alpha} \sigma$ of order $\alpha>0$ with $a \geq 0$ are defined by

$$
\begin{aligned}
& \mathcal{I}_{a+, k}^{\alpha} \sigma(x)=\frac{1}{k \Gamma_{k}(\alpha)} \int_{a}^{x}(x-t)^{\frac{\alpha}{k}-1} \sigma(t) d t, \quad x>a, \\
& \mathcal{I}_{b-, k}^{\alpha} \sigma(x)=\frac{1}{k \Gamma_{k}(\alpha)} \int_{x}^{b}(t-x)^{\frac{\alpha}{k}-1} \sigma(t) d t, \quad x<b,
\end{aligned}
$$

where

$$
\Gamma_{k}(\alpha)=\int_{0}^{\infty} t^{\alpha-1} e^{-\frac{t^{k}}{k}} d t, \quad \operatorname{Re}(\alpha)>0 .
$$

Hadamard fractional integrals of a function with two variables can be given as follows:

Definition 1.3. [19 Let $\sigma \in \mathcal{L}_{1}([a, b] \times[c, d])$. The $k$-Riemann-Liouville fractional integrals ${ }_{k} \mathcal{I}_{a+, c+}^{\alpha} \sigma,{ }_{k} \mathcal{I}_{a+, d-}^{\alpha, \beta} \sigma,{ }_{k} \mathcal{I}_{b-, c+}^{\alpha, \beta} \sigma$ and ${ }_{k} \mathcal{I}_{b-, d_{-}}^{\alpha, \beta} \sigma$ of order $\alpha, \beta, k>0$ with $a, c \geq 0$ are defined by

$$
\begin{aligned}
& { }_{k} \mathcal{I}_{a+, c+}^{\alpha} \sigma(x, y)=\frac{1}{k^{2} \Gamma_{k}(\alpha) \Gamma_{k}(\beta)} \int_{a}^{x} \int_{c}^{y}(x-t)^{\frac{\alpha}{k}-1}(y-s)^{\frac{\beta}{k}-1} \sigma(t, s) d s d t, \\
& x>a, y>c \\
& { }_{k} \mathcal{I}_{a+, d-}^{\alpha, \beta} \sigma(x, y)=\frac{1}{k^{2} \Gamma_{k}(\alpha) \Gamma_{k}(\beta)} \int_{a}^{x} \int_{y}^{d}(x-t)^{\frac{\alpha}{k}-1}(s-y)^{\frac{\beta}{k}-1} \sigma(t, s) d s d t, \\
& x>a, y<d,
\end{aligned}
$$




$$
\begin{aligned}
& { }_{k} \mathcal{I}_{b-, c+}^{\alpha, \beta} \sigma(x, y)=\frac{1}{k^{2} \Gamma_{k}(\alpha) \Gamma_{k}(\beta)} \int_{x}^{b} \int_{c}^{y}(t-x)^{\frac{\alpha}{k}-1}(y-s)^{\frac{\beta}{k}-1} \sigma(t, s) d s d t, \\
& x<b, y>c
\end{aligned}
$$

and

$$
\begin{aligned}
& { }_{k} \mathcal{I}_{b-, d_{-}-}^{\alpha, \beta} \sigma(x, y)=\frac{1}{k^{2} \Gamma_{k}(\alpha) \Gamma_{k}(\beta)} \int_{x}^{b} \int_{y}^{d}(t-x)^{\frac{\alpha}{k}-1}(s-y)^{\frac{\beta}{k}-1} \sigma(t, s) d s d t, \\
& x<b, y<d,
\end{aligned}
$$

respectively.

For more details about Riemann-Liouville fractional integrals, see [20, 22, 24, 27.

Moreover, one can find some recent Hermite-Hadamard inequalities for function of one and two variables via Riemann-Liouville fractional integrals, see [1, 8, 18, 21, 23, 25], [28,- 40].

In [29], Sarikaya et al. define a new left-sided and right-sided generalized fractional integrals as follows:

$$
{ }_{a^{+}} \mathcal{I}_{\phi} \sigma(x)=\int_{a}^{x} \frac{\phi(x-t)}{x-t} \sigma(t) d t, \quad x>a
$$

and

$$
{ }_{b^{-}} \mathcal{I}_{\phi} \sigma(x)=\int_{x}^{b} \frac{\phi(t-x)}{t-x} \sigma(t) d t, \quad x<b,
$$

respectively, where $\phi:[0,+\infty) \longrightarrow[0,+\infty)$ is a function which satisfies

$$
\int_{0}^{1} \frac{\phi(t)}{t} d t<+\infty
$$

Furthermore, they noticed that these generalized fractional integrals may contain some types of fractional integrals such as Riemann-Liouville fractional integral, $k$-Riemann-Liouville fractional integral, Katugampola fractional integral, conformable fractional integral, etc., with some special choices. Inspired by this definition, Yildirim et al. in [35] give the following definitions:

Definition 1.4. Let $\sigma \in \mathcal{L}_{1}([a, b] \times[c, d])$. The Generalized Riemann-Liouville

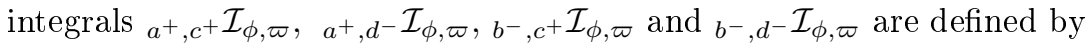

$$
{ }_{a^{+}, c^{+}} \mathcal{I}_{\phi, \varpi} \sigma(x, y)=\int_{a}^{x} \int_{c}^{y} \frac{\phi(x-t)}{x-t} \frac{\varpi(y-s)}{y-s} \sigma(t, s) d s d t, \quad x>a, y>c,
$$




$$
\begin{aligned}
& { }_{a^{+}, d^{-}} \mathcal{I}_{\phi, \varpi} \sigma(x, y)=\int_{a}^{x} \int_{y}^{d} \frac{\phi(x-t)}{x-t} \frac{\varpi(s-y)}{s-y} \sigma(t, s) d s d t, \quad x>a, y<d, \\
& b^{-}, c^{+} \\
& \mathcal{I}_{\phi, \varpi} \sigma(x, y)=\int_{x}^{d} \int_{c}^{y} \frac{\phi(t-x)}{t-x} \frac{\varpi(y-s)}{y-s} \sigma(t, s) d s d t, \quad x<b, y>c
\end{aligned}
$$

and

$$
{ }_{b^{-}, d^{-}} \mathcal{I}_{\phi, \varpi} \sigma(x, y)=\int_{x}^{d} \int_{y}^{d} \frac{\phi(t-x)}{t-x} \frac{\varpi(s-y)}{s-y} \sigma(t, s) d s d t, \quad x<b, y<d,
$$

where $\phi, \varpi:[0,+\infty) \longrightarrow[0,+\infty)$ are functions which satisfy $\int_{0}^{1} \frac{\phi(t)}{t} d t<+\infty$ and $\int_{0}^{1} \frac{\varpi(s)}{s} d s<+\infty$, respectively.

Functions of bounded variation of one variable are of great interest and usefulness and they have been much studied because of their valuable properties, such as particularly with respect to additivity, decomposability into monotone functions, continuity, differentiability, measurability, integrability and so on. There are many of papers on inequalities for functions of bounded variation of one variable, see [2]-[4], [9],[14-[16]. Moreover, Dragomir obtained some fractional inequalities involving functions of bounded variation, see [11]-[13].

Functions of bounded variation with two variables are defined as follows:

Definition 1.5. [10] Assume that $\sigma(x, y)$ is defined over the rectangle $Q=[a, b] \times$ $[c, d]$. Let $P$ be a partition of $Q$ with

$$
P: a=x_{0}<x_{1}<\ldots<x_{n}=b, \text { and } c=y_{0}<y_{1}<\ldots<y_{m}=d ;
$$

and for all $i, j$ let

$$
\Delta_{11} \sigma\left(x_{i}, y_{j}\right)=\sigma\left(x_{i-1}, y_{j-1}\right)-\sigma\left(x_{i-1}, y_{j}\right)-\sigma\left(x_{i}, y_{j-1}\right)+\sigma\left(x_{i}, y_{j}\right) .
$$

The function $\sigma(x, y)$ is said to be of bounded variation if the sum

$$
\sum_{i=0}^{n-1} \sum_{j=0}^{m-1}\left|\Delta_{11} \sigma\left(x_{i}, y_{j}\right)\right|
$$

is bounded for all nets.

Therefore, one can define the concept of total variation of a function of variables, as follows.

Let $\sigma$ be of bounded variation on $Q=[a, b] \times[c, d]$, and let $\sum(P)$ denote the sum $\sum_{i=1}^{n} \sum_{j=1}^{m}\left|\Delta_{11} \sigma\left(x_{i}, y_{j}\right)\right|$ corresponding to the partition $P$ of $Q$. The number 


$$
\bigvee_{Q}(\sigma):=\bigvee_{a}^{b} \bigvee_{c}^{d}(\sigma):=\sup \left\{\sum(P): P \in P(Q)\right\}
$$

is called the total variation of $\sigma$ on $Q$.

There are also a few papers on fractional inequalities for functions of bounded variation with two variables, see [5]- 7 . However, there is a few papers fractional integral inequalities for functions of bounded variation with two variables. The

aim of this paper is to establish some fractional Ostrowski and Midpoint type inequalities for functions of bounded variation with two variables. The remainder paper is organized as follows: In Section 2, we present some identities for generalized fractional integrals. In Section 3, some Ostrowski type inequalities for generalized fractional integrals will be obtained for functions of bounded variation with two variables. In Section 4 we give some inequalities for $k$-Riemann-Liouville fractional integrals as special cases of our main results. In Section 5 a brief conclusion is provided as well.

\section{Some identities for Generalized Fractional Integrals}

Firstly, we define the following functions which will be used throughout this paper:

$$
\begin{aligned}
& \Phi_{1}(x)=\int_{a}^{x} \frac{\phi(x-t)}{x-t} d t, \quad x>a, \quad \Phi_{2}(x)=\int_{x}^{b} \frac{\phi(t-x)}{t-x} d t, \quad x<b, \\
& \Phi_{3}(x)=\int_{x}^{b} \frac{\phi(b-t)}{b-t} d t, \quad x<b, \quad \Phi_{4}(x)=\int_{a}^{x} \frac{\phi(t-a)}{t-a} d t, \quad x>a,
\end{aligned}
$$

and

$$
\begin{aligned}
& \Psi_{1}(y)=\int_{c}^{y} \frac{\varpi(y-s)}{y-s} d s, \quad y>c, \quad \Psi_{2}(y)=\int_{y}^{d} \frac{\varpi(s-y)}{s-y} d s, \quad y<d, \\
& \Psi_{3}(y)=\int_{y}^{d} \frac{\varpi(d-s)}{d-s} d s, \quad y<d, \quad \Psi_{4}(y)=\int_{c}^{y} \frac{\varpi(s-c)}{s-c} d s, \quad y>c .
\end{aligned}
$$

Here we have $\Phi_{3}(a)=\Phi_{4}(b)$ and $\Psi_{3}(c)=\Psi_{4}(d)$.

Now we prove the following equalities:

Lemma 1. If $\sigma: \Delta=[a, b] \times[c, d] \rightarrow \Re$ is integrable on $\Delta$, then we have the following identity:

$$
\begin{aligned}
& \sigma(x, y)-\frac{1}{\Omega(x)}\left[{ }_{a^{+}} \mathcal{I}_{\phi} \sigma(x, y)+{ }_{{ }^{-}} \mathcal{I}_{\phi} \sigma(x, y)\right]-\frac{1}{\Lambda(y)}\left[{ }_{c^{+}} \mathcal{I}_{\varpi} \sigma(x, y)+{ }_{d^{-}} \mathcal{I}_{\varpi} \sigma(x, y)\right] \\
+ & \frac{1}{\Omega(x) \Lambda(y)} \times
\end{aligned}
$$




$$
\begin{gathered}
\times\left[{ }_{a^{+}, c^{+}} \mathcal{I}_{\phi, \varpi} \sigma(x, y)+{ }_{a^{+}, d^{-}} \mathcal{I}_{\phi, \varpi} \sigma(x, y)+{ }_{b^{-}, c^{+}} \mathcal{I}_{\phi, \varpi} \sigma(x, y)+{ }_{{ }^{-}, d^{-}} \mathcal{I}_{\phi, \varpi} \sigma(x, y)\right] \\
=\frac{1}{\Omega(x) \Lambda(y)} \sum_{k=1}^{4} \mathcal{I}_{k}
\end{gathered}
$$

where

$$
\begin{aligned}
& \mathcal{I}_{1}=\int_{a}^{x} \int_{c}^{y} \frac{\phi(x-t)}{x-t} \frac{\varpi(y-s)}{y-s}[\sigma(t, s)-\sigma(t, y)-\sigma(x, s)+\sigma(x, y)] d s d t, \\
& \mathcal{I}_{2}=\int_{a}^{x} \int_{y}^{d} \frac{\phi(x-t)}{x-t} \frac{\varpi(s-y)}{s-y}[\sigma(t, s)-\sigma(t, y)-\sigma(x, s)+\sigma(x, y)] d s d t, \\
& \mathcal{I}_{3}=\int_{x}^{b} \int_{c}^{y} \frac{\phi(t-x)}{t-x} \frac{\varpi(y-s)}{y-s}[\sigma(t, s)-\sigma(t, y)-\sigma(x, s)+\sigma(x, y)] d s d t, \\
& \mathcal{I}_{4}=\int_{x}^{b} \int_{y}^{d} \frac{\phi(t-x)}{t-x} \frac{\varpi(s-y)}{s-y}[\sigma(t, s)-\sigma(t, y)-\sigma(x, s)+\sigma(x, y)] d s d t
\end{aligned}
$$

and

$$
\Omega(x)=\Phi_{1}(x)+\Phi_{2}(x), \quad \Lambda(y)=\Psi_{1}(y)+\Psi_{2}(y)
$$

are defined from 2.1) and 2.3, for all $(x, y) \in \Delta$.

Proof. By the Definition 1.4 we have

$$
\begin{gathered}
\mathcal{I}_{1}=\int_{a}^{x} \int_{c}^{y} \frac{\phi(x-t)}{x-t} \frac{\varpi(y-s)}{y-s}[\sigma(t, s)-\sigma(t, y)-\sigma(x, s)+\sigma(x, y)] d s d t \\
={ }_{a^{+}, c^{+}} \mathcal{I}_{\phi, \varpi} \sigma(x, y)-\Psi_{1}(y)_{a^{+}} \mathcal{I}_{\phi} \sigma(x, y)-\Phi_{1}(x){ }_{c^{+}} \mathcal{I}_{\varpi} \sigma(x, y)+ \\
+\Phi_{1}(x) \Psi_{1}(y) \sigma(x, y) .
\end{gathered}
$$

Similarly, we get

$$
\begin{aligned}
\mathcal{I}_{2}={ }_{a^{+}, d^{-}} \mathcal{I}_{\phi, \varpi} \sigma(x, y)-\Psi_{2}(y)_{a^{+}} \mathcal{I}_{\phi} \sigma(x, y)- & \Phi_{1}(x)_{d^{-}} \mathcal{I}_{\varpi} \sigma(x, y)+ \\
& +\Phi_{1}(x) \Psi_{2}(y) \sigma(x, y), \\
\mathcal{I}_{3}=_{b^{-}, c^{+}} \mathcal{I}_{\phi, \varpi} \sigma(x, y)-\Psi_{1}(y)_{b^{-}} \mathcal{I}_{\phi} \sigma(x, y)- & \Phi_{2}(x)_{c^{+}} \mathcal{I}_{\varpi} \sigma(x, y)+ \\
& +\Phi_{2}(x) \Psi_{1}(y) \sigma(x, y)
\end{aligned}
$$

and 


$$
\begin{array}{r}
\mathcal{I}_{4}={ }_{b^{-}, d^{-}} \mathcal{I}_{\phi, \varpi} \sigma(x, y)-\Psi_{2}(y)_{b^{-}} \mathcal{I}_{\phi} \sigma(x, y)- \\
\Phi_{2}(x)_{d^{-}} \mathcal{I}_{\varpi} \sigma(x, y)+ \\
+\Phi_{2}(x) \Psi_{2}(y) \sigma(x, y) .
\end{array}
$$

If we add the equalities $(2.6)-(2.9)$ and then divide the result by $\Omega(x) \Lambda(y)$, we obtain the required identity $(2.5)$.

Lemma 2. If $\sigma: \Delta=[a, b] \times[c, d] \rightarrow \Re$ is integrable on $\Delta$, then we have the following identity:

$$
\begin{gathered}
\sigma(x, y)-\frac{1}{\Omega^{*}(x)}\left[{ }_{x^{+}} \mathcal{I}_{\phi} \sigma(b, y)+{ }_{x^{-}} \mathcal{I}_{\phi} \sigma(a, y)\right] \\
-\frac{1}{\Lambda^{*}(y)}\left[{ }_{y^{+}} \mathcal{I}_{\varpi} \sigma(x, d)+{ }_{y^{-}} \mathcal{I}_{\varpi} \sigma(x, c)\right]+\frac{1}{\Omega^{*}(x) \Lambda^{*}(y)} \times \\
\times\left[{ }_{x^{+}, y^{+}} \mathcal{I}_{\phi, \varpi} \sigma(b, d)+{ }_{x^{+}, y^{-}} \mathcal{I}_{\phi, \varpi} \sigma(b, c)+{ }_{x^{-}, y^{+}} \mathcal{I}_{\phi, \varpi} \sigma(a, d)+{ }_{x^{-}, y^{-}} \mathcal{I}_{\phi, \varpi} \sigma(a, c)\right] \\
=\frac{1}{\Omega^{*}(x) \Lambda^{*}(y)} \sum_{k=5}^{8} \mathcal{I}_{k}
\end{gathered}
$$

where

$$
\begin{aligned}
& \mathcal{I}_{5}=\int_{x}^{b} \int_{y}^{d} \frac{\phi(b-t)}{b-t} \frac{\varpi(d-s)}{d-s}[\sigma(t, s)-\sigma(t, y)-\sigma(x, s)+\sigma(x, y)] d s d t, \\
& \mathcal{I}_{6}=\int_{x}^{b} \int_{c}^{y} \frac{\phi(b-t)}{b-t} \frac{\varpi(s-c)}{s-c}[\sigma(t, s)-\sigma(t, y)-\sigma(x, s)+\sigma(x, y)] d s d t, \\
& \mathcal{I}_{7}=\int_{a}^{x} \int_{y}^{d} \frac{\phi(t-a)}{t-a} \frac{\varpi(d-s)}{d-s}[\sigma(t, s)-\sigma(t, y)-\sigma(x, s)+\sigma(x, y)] d s d t, \\
& \mathcal{I}_{8}=\int_{a}^{x} \int_{c}^{y} \frac{\phi(t-a)}{t-a} \frac{\varpi(s-c)}{s-c}[\sigma(t, s)-\sigma(t, y)-\sigma(x, s)+\sigma(x, y)] d s d t
\end{aligned}
$$

and

$$
\Omega^{*}(x)=\Phi_{3}(x)+\Phi_{4}(x), \quad \Lambda^{*}(y)=\Psi_{3}(y)+\Psi_{4}(y)
$$

are defined in (2.2) and (2.4), for all $(x, y) \in \Delta$.

Proof. By the Definition 1.4 we have

$$
\mathcal{I}_{5}=\int_{x}^{b} \int_{y}^{d} \frac{\phi(b-t)}{b-t} \frac{\varpi(d-s)}{d-s}[\sigma(t, s)-\sigma(t, y)-\sigma(x, s)+\sigma(x, y)] d s d t
$$


$={ }_{x^{+}, y^{+}} \mathcal{I}_{\phi, \varpi} \sigma(b, d)-\Psi_{3}(y)_{x^{+}} \mathcal{I}_{\phi} \sigma(b, y)-\Phi_{3}(x){ }_{y^{+}} \mathcal{I}_{\varpi} \sigma(x, d)+\Phi_{3}(x) \Psi_{3}(y) \sigma(x, y)$,

and similarly

$$
\begin{array}{r}
\mathcal{I}_{6}={ }_{x^{+}, y^{-}} \mathcal{I}_{\phi, \varpi} \sigma(b, c)-\Psi_{4}(y)_{x^{+}} \mathcal{I}_{\phi} \sigma(b, y)-\Phi_{3}(x)_{y^{-}} \mathcal{I}_{\varpi} \sigma(x, c)+ \\
\Phi_{3}(x) \Psi_{4}(y) \sigma(x, y), \\
\mathcal{I}_{7}={ }_{x^{-}, y^{+}} \mathcal{I}_{\phi, \varpi} \sigma(a, d)-\Psi_{3}(y)_{x^{-}} \mathcal{I}_{\phi} \sigma(a, y)-\Phi_{4}(x)_{y^{+}} \mathcal{I}_{\varpi} \sigma(x, d)+ \\
\Phi_{4}(x) \Psi_{3}(y) \sigma(x, y)
\end{array}
$$

and

$$
\begin{array}{r}
\mathcal{I}_{8}={ }_{x^{-}, y^{-}} \mathcal{I}_{\phi, \varpi} \sigma(a, c)-\Psi_{4}(y)_{x^{-}} \mathcal{I}_{\phi} \sigma(a, y)-\Phi_{4}(x)_{y^{-}} \mathcal{I}_{\varpi} \sigma(x, c)+ \\
\Phi_{4}(x) \Psi_{4}(y) \sigma(x, y) .
\end{array}
$$

If we add the equalities $2.11-(2.15)$ and then divide the result by $\Omega^{*}(x) \Lambda^{*}(y)$, we obtain the required identity (2.10).

Lemma 3. If $\sigma: \Delta=[a, b] \times[c, d] \rightarrow \Re$ is integrable on $\Delta$, then we have the following identity:

$$
\begin{aligned}
& \frac{\sigma(a, c)+\sigma(a, d)+\sigma(b, c)+\sigma(b, d)}{4} \Phi_{3}(a) \Psi_{3}(c) \\
& -\frac{\Phi_{3}(a)}{4}\left[{ }_{c^{+}} \mathcal{I}_{\varpi} \sigma(b, d)+{ }_{d^{-}} \mathcal{I}_{\varpi} \sigma(b, c)+{ }_{c^{+}} \mathcal{I}_{\varpi} \sigma(a, d)+{ }_{d^{-}} \mathcal{I}_{\varpi} \sigma(a, c)\right] \\
& -\frac{\Psi_{3}(c)}{4}\left[{ }_{a^{+}} \mathcal{I}_{\phi} \sigma(b, d)+{ }_{b^{-}} \mathcal{I}_{\phi} \sigma(a, d)+{ }_{a^{+}} \mathcal{I}_{\phi} \sigma(b, c)+{ }_{b^{-}} \mathcal{I}_{\phi} \sigma(a, c)\right] \\
& +\frac{1}{4}\left[{ }_{a^{+}, c^{+}} \mathcal{I}_{\phi, \varpi} \sigma(b, d)+{ }_{a^{+}, d^{-}} \mathcal{I}_{\phi, \varpi} \sigma(b, c)+{ }_{b^{-}, c^{+}} \mathcal{I}_{\phi, \varpi} \sigma(a, d)+{ }_{b^{-}, d^{-}} \mathcal{I}_{\phi, \varpi} \sigma(a, c)\right] \\
= & \frac{1}{4} \sum_{k=9}^{12} \mathcal{I}_{k},
\end{aligned}
$$

where

$$
\begin{aligned}
& \mathcal{I}_{9}=\int_{a}^{b} \int_{c}^{d} \frac{\phi(b-t)}{b-t} \frac{\varpi(d-s)}{d-s}[\sigma(t, s)-\sigma(t, d)-\sigma(b, s)+\sigma(b, d)] d s d t, \\
& \mathcal{I}_{10}=\int_{a}^{b} \int_{c}^{d} \frac{\phi(b-t)}{b-t} \frac{\varpi(s-c)}{s-c}[\sigma(t, s)-\sigma(t, c)-\sigma(b, s)+\sigma(b, c)] d s d t, \\
& \mathcal{I}_{11}=\int_{a}^{b} \int_{c}^{d} \frac{\phi(t-a)}{t-a} \frac{\varpi(d-s)}{d-s}[\sigma(t, s)-\sigma(t, d)-\sigma(a, s)+\sigma(a, d)] d s d t,
\end{aligned}
$$




$$
\mathcal{I}_{12}=\int_{a}^{b} \int_{c}^{d} \frac{\phi(t-a)}{t-a} \frac{\varpi(s-c)}{s-c}[\sigma(t, s)-\sigma(t, c)-\sigma(a, s)+\sigma(a, c)] d s d t
$$

for all $(x, y) \in \Delta$.

Proof. By the Definition 1.4 we have

$$
\begin{array}{r}
\mathcal{I}_{9}=\int_{a}^{b} \int_{c}^{d} \frac{\phi(b-t)}{b-t} \frac{\varpi(d-s)}{d-s}[\sigma(t, s)-\sigma(t, d)-\sigma(b, s)+\sigma(b, d)] d s d t \\
={ }_{a^{+}, c^{+}} \mathcal{I}_{\phi, \varpi} \sigma(b, d)-\Psi_{3}(c)_{a^{+}} \mathcal{I}_{\phi} \sigma(b, d)-\Phi_{3}(a)_{c^{+}} \mathcal{I}_{\varpi} \sigma(b, d)+ \\
\Phi_{3}(a) \Psi_{3}(c) \sigma(b, d),
\end{array}
$$

and similarly

$$
\begin{array}{r}
\mathcal{I}_{10}={ }_{a^{+}, d^{-}} \mathcal{I}_{\phi, \varpi} \sigma(b, c)-\Psi_{4}(d)_{a^{+}} \mathcal{I}_{\phi} \sigma(b, c)-\Phi_{3}(a)_{d^{-}} \mathcal{I}_{\varpi} \sigma(b, c)+ \\
\Phi_{3}(a) \Psi_{4}(d) \sigma(b, c), \\
\mathcal{I}_{11}={ }_{b^{-}, c^{+}} \mathcal{I}_{\phi, \varpi} \sigma(a, d)-\Psi_{3}(c)_{b^{-}} \mathcal{I}_{\phi} \sigma(a, d)-\Phi_{4}(b)_{c^{+}} \mathcal{I}_{\varpi} \sigma(a, d)+ \\
\Phi_{4}(b) \Psi_{3}(c) \sigma(a, d)
\end{array}
$$

and

$$
\begin{array}{r}
\mathcal{I}_{12}={ }_{b^{-}, d^{-}} \mathcal{I}_{\phi, \varpi} \sigma(a, c)-\Psi_{4}(d)_{b^{-}} \mathcal{I}_{\phi} \sigma(a, c)-\Phi_{4}(b)_{d^{-}} \mathcal{I}_{\varpi} \sigma(a, c)+ \\
\Phi_{4}(b) \Psi_{4}(d) \sigma(a, c) .
\end{array}
$$

If we add the equalities $2.16-2.19$ and then divide the result by 4 , we obtain the required identity 3.8 .

\section{Some Ostrowski type inequalities for Generalized Fractional \\ INTEGRALS}

In this section, Ostrowski type inequalities involving generalized fractional integrals are obtained for functions of bounded variation with two variables.

Theorem 2. If $\sigma: \Delta \rightarrow \Re$ is of bounded variation on $\Delta$, then we have the following inequalities:

$\mid \sigma(x, y)-\frac{1}{\Omega(x)}\left[{ }_{a^{+}} \mathcal{I}_{\phi} \sigma(x, y)+{ }_{b^{-}} \mathcal{I}_{\phi} \sigma(x, y)\right]-\frac{1}{\Lambda(y)}\left[{ }_{c^{+}} \mathcal{I}_{\varpi} \sigma(x, y)+{ }_{d^{-}} \mathcal{I}_{\varpi} \sigma(x, y)\right]+$ 


$$
\begin{aligned}
& +\frac{1}{\Omega(x) \Lambda(y)} \times\left[{ }_{a^{+}, c^{+}} \mathcal{I}_{\phi, \varpi} \sigma(x, y)+{ }_{a^{+}, d^{-}} \mathcal{I}_{\phi, \varpi} \sigma(x, y)+{ }_{b^{-}, c^{+}} \mathcal{I}_{\phi, \varpi} \sigma(x, y)+\right. \\
& \left.+{ }_{b^{-}, d^{-}} \mathcal{I}_{\phi, \varpi} \sigma(x, y)\right] \\
& \leq \frac{1}{\Omega(x) \Lambda(y)}\left[\int_{a}^{x} \int_{c}^{y} \frac{\phi(x-t)}{x-t} \frac{\varpi(y-s)}{y-s} \bigvee_{t}^{x} \bigvee_{s}^{y}(\sigma) d s d t\right. \\
& +\int_{a}^{x} \int_{y}^{d} \frac{\phi(x-t)}{x-t} \frac{\varpi(s-y)}{s-y} \bigvee_{t}^{x} \bigvee_{y}^{s}(\sigma) d s d t \\
& \left.+\int_{x}^{b} \int_{c}^{y} \frac{\phi(t-x)}{t-x} \frac{\varpi(y-s)}{y-s} \bigvee_{x}^{t} \bigvee_{s}^{y}(\sigma) d s d t+\int_{x}^{b} \int_{y}^{d} \frac{\phi(t-x)}{t-x} \frac{\varpi(s-y)}{s-y} \bigvee_{x}^{t} \bigvee_{y}^{s}(\sigma) d s d t\right] \\
& \leq \frac{1}{\Omega(x) \Lambda(y)}\left[\Phi_{1}(x) \Psi_{1}(y) \bigvee_{a}^{x} \bigvee_{c}^{y}(\sigma)+\Phi_{1}(x) \Psi_{2}(y) \bigvee_{a}^{x} \bigvee_{y}^{d}(\sigma)+\Phi_{2}(x) \Psi_{1}(y) \bigvee_{x}^{b} \bigvee_{c}^{y}(\sigma)\right. \\
& \left.+\Phi_{2}(x) \Psi_{2}(y) \bigvee_{x}^{b} \bigvee_{y}^{d}(\sigma)\right] \\
& \leq \frac{1}{\Omega(x) \Lambda(y)} \max \left\{\Phi_{1}(x), \Phi_{2}(x)\right\} \max \left\{\Psi_{1}(y), \Psi_{2}(y)\right\} \bigvee_{a}^{b} \bigvee_{c}^{d}(\sigma)
\end{aligned}
$$

for all $(x, y) \in \Delta$.

Proof. By using modulus in the Lemma 1, we get

$$
\begin{aligned}
& \mid \sigma(x, y)-\frac{1}{\Omega(x)}\left[{ }_{a}+\mathcal{I}_{\phi} \sigma(x, y)+{ }_{b^{-}} \mathcal{I}_{\phi} \sigma(x, y)\right]-\frac{1}{\Lambda(y)}\left[{ }_{c^{+}} \mathcal{I}_{\varpi} \sigma(x, y)+{ }_{d^{-}} \mathcal{I}_{\varpi} \sigma(x, y)\right] \\
& +\frac{1}{\Omega(x) \Lambda(y)} \\
& \times\left[{ }_{a^{+}, c^{+}} \mathcal{I}_{\phi, \varpi} \sigma(x, y)+{ }_{a^{+}, d^{-}} \mathcal{I}_{\phi, \varpi} \sigma(x, y)+{ }_{b^{-}, c^{+}} \mathcal{I}_{\phi, \varpi} \sigma(x, y)+{ }_{b^{-}, d^{-}} \mathcal{I}_{\phi, \varpi} \sigma(x, y)\right] \mid \\
& \leq \frac{1}{\Omega(x) \Lambda(y)} \times\left[\int_{a}^{x} \int_{c}^{y} \frac{\phi(x-t)}{x-t} \frac{\varpi(y-s)}{y-s}|\sigma(t, s)-\sigma(t, y)-\sigma(x, s)+\sigma(x, y)| d s d t\right. \\
& +\int_{a}^{x} \int_{y}^{d} \frac{\phi(x-t)}{x-t} \frac{\varpi(s-y)}{s-y}|\sigma(t, s)-\sigma(t, y)-\sigma(x, s)+\sigma(x, y)| d s d t
\end{aligned}
$$




$$
\begin{aligned}
& +\int_{x}^{b} \int_{c}^{y} \frac{\phi(t-x)}{t-x} \frac{\varpi(y-s)}{y-s}|\sigma(t, s)-\sigma(t, y)-\sigma(x, s)+\sigma(x, y)| d s d t \\
& \left.+\int_{x}^{b} \int_{y}^{d} \frac{\phi(t-x)}{t-x} \frac{\varpi(s-y)}{s-y}|\sigma(t, s)-\sigma(t, y)-\sigma(x, s)+\sigma(x, y)| d s d t\right] \\
& =A(x, y) .
\end{aligned}
$$

Since $\sigma$ is of bounded variation on $\Delta$, we have the following inequalities

$$
\begin{aligned}
& \mid \sigma(t, s)-\sigma(t, y)-\sigma(x, s)+\sigma(x, y) \mid \leq \bigvee_{t}^{x} \bigvee_{s}^{y}(\sigma) \leq \\
& \leq \bigvee_{a}^{x} \bigvee_{c}^{y}(\sigma) \text { for }(t, s) \in[a, x) \times[c, y), \\
&|\sigma(t, s)-\sigma(t, y)-\sigma(x, s)+\sigma(x, y)| \leq \bigvee_{t}^{x} \bigvee_{y}^{s}(\sigma) \leq \\
& \leq \bigvee_{a}^{x} \bigvee_{y}^{d}(\sigma) \text { for }(t, s) \in[a, x) \times[y, d], \\
&|\sigma(t, s)-\sigma(t, y)-\sigma(x, s)+\sigma(x, y)| \leq \bigvee_{x}^{t} \bigvee_{s}^{y}(\sigma) \leq \\
& \leq \bigvee_{x}^{b} \bigvee_{c}^{y}(\sigma) \text { for }(t, s) \in[x, b] \times[c, y)
\end{aligned}
$$

and

$$
\begin{gathered}
|\sigma(t, s)-\sigma(t, y)-\sigma(x, s)+\sigma(x, y)| \leq \bigvee_{x}^{t} \bigvee_{y}^{s}(\sigma) \leq \\
\leq \bigvee_{x}^{b} \bigvee_{y}^{d}(\sigma) \text { for }(t, s) \in[x, b] \times[y, d] .
\end{gathered}
$$

By substituting the inequalities $(3.3)-(3.6)$ in $(3.2)$, we obtain

$$
\begin{aligned}
& A(x, y) \leq \frac{1}{\Omega(x) \Lambda(y)} \times\left[\int_{a}^{x} \int_{c}^{y} \frac{\phi(x-t)}{x-t} \frac{\varpi(y-s)}{y-s} \bigvee_{t}^{x} \bigvee_{s}^{y}(\sigma) d s d t\right. \\
& +\int_{a}^{x} \int_{y}^{d} \frac{\phi(x-t)}{x-t} \frac{\varpi(s-y)}{s-y} \bigvee_{t}^{x} \bigvee_{y}^{s}(\sigma) d s d t
\end{aligned}
$$




$$
\begin{aligned}
& +\int_{x}^{b} \int_{c}^{y} \frac{\phi(t-x)}{t-x} \frac{\varpi(y-s)}{y-s} \bigvee_{x}^{t} \bigvee_{s}^{y}(\sigma) d s d t \\
& \left.+\int_{x}^{b} \int_{y}^{d} \frac{\phi(t-x)}{t-x} \frac{\varpi(s-y)}{s-y} \bigvee_{x}^{t} \bigvee_{y}^{s}(\sigma) d s d t\right] \\
& \leq \frac{1}{\Omega(x) \Lambda(y)}\left[\Phi_{1}(x) \Psi_{1}(y) \bigvee_{a}^{x} \bigvee_{c}^{y}(\sigma)+\Phi_{1}(x) \Psi_{2}(y) \bigvee_{a}^{x} \bigvee_{y}^{d}(\sigma)\right. \\
& \left.+\Phi_{2}(x) \Psi_{1}(y) \bigvee_{x}^{b} \bigvee_{c}^{y}(\sigma)+\Phi_{2}(x) \Psi_{2}(y) \bigvee_{x}^{b} \bigvee_{y}^{d}(\sigma)\right]
\end{aligned}
$$

This completes the proof of the first and second inequality in (3.1). The last inequality is obvious from the maximum properties. The proof of Theorem 2 is completed.

Theorem 3. If $\sigma: \Delta \rightarrow \Re$ is of bounded variation on $\Delta$, then we have the following inequalities:

$$
\begin{aligned}
& \mid \sigma(x, y)-\frac{1}{\Omega^{*}(x)}\left[{ }_{x^{+}} \mathcal{I}_{\phi} \sigma(b, y)+{ }_{x^{-}} \mathcal{I}_{\phi} \sigma(a, y)\right]- \\
& -\frac{1}{\Lambda^{*}(y)}\left[{ }_{y^{+}} \mathcal{I}_{\varpi} \sigma(x, d)+{ }_{y^{-}} \mathcal{I}_{\varpi} \sigma(x, c)\right]+\frac{1}{\Omega^{*}(x) \Lambda^{*}(y)} \times \\
& \times\left[{ }_{x^{+}, y^{+}} \mathcal{I}_{\phi, \varpi} \sigma(b, d)+{ }_{x^{+}, y^{-}} \mathcal{I}_{\phi, \varpi} \sigma(b, c)+{ }_{x^{-}, y^{+}} \mathcal{I}_{\phi, \varpi} \sigma(a, d)+{ }_{x^{-}, y^{-}} \mathcal{I}_{\phi, \varpi} \sigma(a, c)\right] \\
& \leq \frac{1}{\Omega^{*}(x) \Lambda^{*}(y)}\left[\int_{x}^{b} \int_{y}^{d} \frac{\phi(b-t)}{b-t} \frac{\varpi(d-s)}{d-s} \bigvee_{x}^{t} \bigvee_{y}^{s}(\sigma) d s d t+\right. \\
& +\int_{x}^{b} \int_{c}^{y} \frac{\phi(b-t)}{b-t} \frac{\varpi(s-c)}{s-c} \bigvee_{x}^{t} \bigvee_{s}^{y}(\sigma) d s d t \\
& \left.+\int_{a}^{x} \int_{y}^{d} \frac{\phi(t-a)}{t-a} \frac{\varpi(d-s)}{d-s} \bigvee_{t}^{x} \bigvee_{y}^{s}(\sigma) d s d t+\int_{a}^{x} \int_{c}^{y} \frac{\phi(t-a)}{t-a} \frac{\varpi(s-c)}{s-c} \bigvee_{t}^{x} \bigvee_{s}^{y}(\sigma) d s d t\right] \\
& \leq \frac{1}{\Omega^{*}(x) \Lambda^{*}(y)}\left[\Phi_{3}(x) \Psi_{3}(y) \bigvee_{x}^{b} \bigvee_{y}^{d}(\sigma)+\Phi_{3}(x) \Psi_{4}(y) \bigvee_{x}^{b} \bigvee_{c}^{y}(\sigma)+\right. \\
& \left.+\Phi_{4}(x) \Psi_{3}(y) \bigvee_{a}^{x} \bigvee_{y}^{d}(\sigma)+\Phi_{4}(x) \Psi_{4}(y) \bigvee_{a}^{x} \bigvee_{c}^{y}(\sigma)\right]
\end{aligned}
$$


ON GENERALIZED FRACTIONAL INTEGRAL INEQUALITIES FOR ...

$$
\leq \frac{1}{\Omega(x) \Lambda(y)} \max \left\{\Phi_{1}(x), \Phi_{2}(x)\right\} \max \left\{\Psi_{1}(y), \Psi_{2}(y)\right\} \bigvee_{a}^{b} \bigvee_{c}^{d}(\sigma)
$$

for all $(x, y) \in \Delta$.

Proof. By using modulus in the Lemma 2, we get

$$
\begin{aligned}
& \mid \sigma(x, y)-\frac{1}{\Omega^{*}(x)}\left[{ }_{x^{+}} \mathcal{I}_{\phi} \sigma(b, y)+{ }_{x^{-}} \mathcal{I}_{\phi} \sigma(a, y)\right]-\frac{1}{\Lambda^{*}(y)}\left[{ }_{y^{+}} \mathcal{I}_{\varpi} \sigma(x, d)+{ }_{y^{-}} \mathcal{I}_{\varpi} \sigma(x, c)\right] \\
& +\frac{1}{\Omega^{*}(x) \Lambda^{*}(y)} \times\left[{ }_{x^{+}, y^{+}} \mathcal{I}_{\phi, \varpi} \sigma(b, d)+{ }_{x^{+}, y^{-}} \mathcal{I}_{\phi, \varpi} \sigma(b, c)+\right. \\
& \left.+{ }_{x^{-}, y^{+}} \mathcal{I}_{\phi, \varpi} \sigma(a, d)+{ }_{x^{-}, y^{-}} \mathcal{I}_{\phi, \varpi} \sigma(a, c)\right] \\
& \leq \frac{1}{\Omega^{*}(x) \Lambda^{*}(y)}\left[\int_{x}^{b} \int_{y}^{d} \frac{\phi(b-t)}{b-t} \frac{\varpi(d-s)}{d-s} \bigvee_{x}^{t} \bigvee_{y}^{s}(\sigma) d s d t+\right. \\
& +\int_{x}^{b} \int_{c}^{y} \frac{\phi(b-t)}{b-t} \frac{\varpi(s-c)}{s-c} \bigvee_{x}^{t} \bigvee_{s}^{y}(\sigma) d s d t \\
& \left.+\int_{a}^{x} \int_{y}^{d} \frac{\phi(t-a)}{t-a} \frac{\varpi(d-s)}{d-s} \bigvee_{t}^{x} \bigvee_{y}^{s}(\sigma) d s+\int_{a}^{x} \int_{c}^{y} \frac{\phi(t-a)}{t-a} \frac{\varpi(s-c)}{s-c} \bigvee_{t}^{x} \bigvee_{s}^{y}(\sigma) d s d t\right] \\
& \leq \frac{1}{\Omega^{*}(x) \Lambda^{*}(y)}\left[\Phi_{3}(x) \Psi_{3}(y) \bigvee_{x}^{b} \bigvee_{y}^{d}(\sigma)+\Phi_{3}(x) \Psi_{4}(y) \bigvee_{x}^{b} \bigvee_{c}^{y}(\sigma)+\right. \\
& \left.+\Phi_{4}(x) \Psi_{3}(y) \bigvee_{a}^{x} \bigvee_{y}^{d}(\sigma)+\Phi_{4}(x) \Psi_{4}(y) \bigvee_{a}^{x} \bigvee_{c}^{y}(\sigma)\right]
\end{aligned}
$$

The proof of Theorem 3 is completed.

Theorem 4. If $\sigma: \Delta \rightarrow \Re$ is of bounded variation on $\Delta$, then we have the following inequalities:

$$
\begin{gathered}
\mid \frac{\sigma(a, c)+\sigma(a, d)+\sigma(b, c)+\sigma(b, d)}{4} \Phi_{3}(a) \Psi_{3}(c) \\
-\frac{\Phi_{3}(a)}{4}\left[{ }_{c^{+}} \mathcal{I}_{\varpi} \sigma(b, d)+{ }_{d^{-}} \mathcal{I}_{\varpi} \sigma(b, c)+{ }_{{ }^{+}} \mathcal{I}_{\varpi} \sigma(a, d)+{ }_{d^{-}} \mathcal{I}_{\varpi} \sigma(a, c)\right] \\
-\frac{\Psi_{3}(c)}{4}\left[{ }_{a^{+}} \mathcal{I}_{\phi} \sigma(b, d)+{ }_{b^{-}} \mathcal{I}_{\phi} \sigma(a, d)+{ }_{a^{+}} \mathcal{I}_{\phi} \sigma(b, c)+{ }_{b^{-}} \mathcal{I}_{\phi} \sigma(a, c)\right] \\
+\frac{1}{4}\left[{ }_{a^{+}, c^{+}} \mathcal{I}_{\phi, \varpi} \sigma(b, d)+{ }_{a^{+}, d^{-}} \mathcal{I}_{\phi, \varpi} \sigma(b, c)+{ }_{b^{-},{ }^{+}} \mathcal{I}_{\phi, \varpi} \sigma(a, d)+{ }_{b^{-}, d^{-}} \mathcal{I}_{\phi, \varpi} \sigma(a, c)\right] \mid
\end{gathered}
$$




$$
\begin{gathered}
\leq \frac{1}{4}\left[\int_{a}^{b} \int_{c}^{d} \frac{\phi(b-t)}{b-t} \frac{\varpi(d-s)}{d-s} \bigvee_{t}^{b} \bigvee_{s}^{d}(\sigma) d s d t+\int_{a}^{b} \int_{c}^{d} \frac{\phi(b-t)}{b-t} \frac{\varpi(s-c)}{s-c} \bigvee_{t}^{b} \bigvee_{c}^{s}(\sigma) d s d t\right. \\
\left.+\int_{a} \int_{c}^{b} \frac{\phi(t-a)}{t-a} \frac{\varpi(d-s)}{d-s} \bigvee_{a}^{t} \bigvee_{s}^{d}(\sigma) d s d t+\int_{a}^{b} \int_{c}^{d} \frac{\phi(t-a)}{t-a} \frac{\varpi(s-c)}{s-c} \bigvee_{a}^{t} \bigvee_{c}^{s}(\sigma) d s d t\right] \\
\leq \Phi_{3}(a) \Psi_{3}(c) \bigvee_{a}^{b} \bigvee_{c}^{d}(\sigma)
\end{gathered}
$$

for all $(x, y) \in \Delta$.

Proof. By using modulus in the Lemma 3, we get

$$
\begin{aligned}
& \mid \frac{\sigma(a, c)+\sigma(a, d)+\sigma(b, c)+\sigma(b, d)}{4} \Phi_{3}(a) \Psi_{3}(c) \\
& -\frac{\Phi_{3}(a)}{4}\left[{ }_{c^{+}} \mathcal{I}_{\varpi} \sigma(b, d)+{ }_{d^{-}} \mathcal{I}_{\varpi} \sigma(b, c)+{ }_{c^{+}} \mathcal{I}_{\varpi} \sigma(a, d)+{ }_{d^{-}} \mathcal{I}_{\varpi} \sigma(a, c)\right] \\
& -\frac{\Psi_{3}(c)}{4}\left[{ }_{a}+\mathcal{I}_{\phi} \sigma(b, d)+{ }_{b^{-}} \mathcal{I}_{\phi} \sigma(a, d)+{ }_{a^{+}} \mathcal{I}_{\phi} \sigma(b, c)+{ }_{b^{-}} \mathcal{I}_{\phi} \sigma(a, c)\right] \\
& +\frac{1}{4}\left[a^{+}, c^{+} \mathcal{I}_{\phi, \varpi} \sigma(b, d)+{ }_{a^{+}, d^{-}} \mathcal{I}_{\phi, \varpi} \sigma(b, c)\right. \\
& \left.+{ }_{b^{-}, c^{+}} \mathcal{I}_{\phi, \varpi} \sigma(a, d)+{ }_{b^{-}, d^{-}} \mathcal{I}_{\phi, \varpi} \sigma(a, c)\right] \mid \\
& \quad \frac{1}{4} \int_{a}^{b} \int_{c}^{d} \frac{\phi(b-t)}{b-t} \frac{\varpi(d-s)}{d-s}|\sigma(t, s)-\sigma(t, d)-\sigma(b, s)+\sigma(b, d)| d s d t(3.9) \\
& +
\end{aligned}
$$

We also have

$$
\int_{a}^{b} \int_{c}^{d} \frac{\phi(b-t)}{b-t} \frac{\varpi(d-s)}{d-s}|\sigma(t, s)-\sigma(t, d)-\sigma(b, s)+\sigma(b, d)| d s d t(3.10)
$$


ON GENERALIZED FRACTIONAL INTEGRAL INEQUALITIES FOR ...

$$
\begin{aligned}
& \leq \int_{a}^{b} \int_{c}^{d} \frac{\phi(b-t)}{b-t} \frac{\varpi(d-s)}{d-s} \bigvee_{t}^{b} \bigvee_{s}^{d}(\sigma) d s d t \\
& \leq \bigvee_{a}^{b} \bigvee_{c}^{d}(\sigma) \int_{a}^{b} \int_{c}^{d} \frac{\phi(b-t)}{b-t} \frac{\varpi(d-s)}{d-s} d s d t \\
& =\Phi_{3}(a) \Psi_{3}(c) \bigvee_{a}^{b} \bigvee_{c}^{d}(\sigma) .
\end{aligned}
$$

Similarly, we obtain

$$
\begin{aligned}
& \int_{a}^{b} \int_{c}^{d} \frac{\phi(b-t)}{b-t} \frac{\varpi(s-c)}{s-c}|\sigma(t, s)-\sigma(t, c)-\sigma(b, s)+\sigma(b, c)| d s d t(3.11) \\
\leq & \int_{a}^{b} \int_{c}^{d} \frac{\phi(b-t)}{b-t} \frac{\varpi(s-c)}{s-c} \bigvee_{t}^{b} \bigvee_{c}^{s}(\sigma) d s d t \\
\leq & \Phi_{3}(a) \Psi_{4}(d) \bigvee_{a}^{b} \bigvee_{c}^{d}(\sigma), \\
\leq & \int_{a}^{b} \int_{c}^{d} \frac{\phi(t-a)}{t-a} \frac{\varpi(d-s)}{d-s}|\sigma(t, s)-\sigma(t, d)-\sigma(a, s)+\sigma(a, d)| d s d t(3.12) \\
\leq & \int_{c}^{d}(b) \Psi_{3}(c) \bigvee \bigvee_{a} \bigvee_{c}^{d}(\sigma) \\
& \int_{b}^{d-a} \frac{\varpi(d-s)}{d-s} \bigvee_{a}^{t} \bigvee_{s}^{d}(\sigma) d s d t
\end{aligned}
$$

and

$$
\begin{aligned}
& \int_{a}^{b} \int_{c}^{d} \frac{\phi(t-a)}{t-a} \frac{\varpi(s-c)}{s-c}|\sigma(t, s)-\sigma(t, c)-\sigma(a, s)+\sigma(a, c)| d s d t(3.13) \\
\leq & \int_{a}^{b} \int_{c}^{d} \frac{\phi(t-a)}{t-a} \frac{\varpi(s-c)}{s-c} \bigvee_{a}^{t} \bigvee_{c}^{s}(\sigma) d s d t \\
\leq & \Phi_{4}(b) \Psi_{4}(d) \bigvee_{a}^{b} \bigvee_{c}^{d}(\sigma) .
\end{aligned}
$$


If we substitute the inequalities $3.10-(3.13)$ in $(3.9)$, we obtain the required inequality 3.8 .

Corollary 4.1. If we take $x=\frac{a+b}{2}$ and $y=\frac{c+d}{2}$ in Theorem 2 and Theorem 3 , then we obtain some midpoint type inequalities, but the details are not presented here.

Remark 3.1: If we choose $\phi(t)=\frac{t^{\alpha}}{\Gamma(\alpha)}, t \in[a, b]$ and $\varpi(s)=\frac{s^{\beta}}{\Gamma(\beta)}, s \in[c, d]$, $\alpha, \beta>0$ in Theorem 2, Theorem 3 and Theorem 4 , then we obtain some Ostrowski type inequalities for Riemann-Liouville fractional integrals which were proved by Erden et al. in [17.

\section{Some inequalities for $k$-Riemann-Liouville Fractional Integrals}

Firstly, we define the following functions:

$$
M(a, b ; x):=(x-a)^{\frac{\alpha}{k}}+(b-x)^{\frac{\alpha}{k}}
$$

and

$$
N(c, d ; y):=(y-c)^{\frac{\beta}{k}}+(d-y)^{\frac{\beta}{k}}
$$

for $(x, y) \in[a, b] \times[c, d]$.

By choosing $\phi(t)=\frac{t^{\frac{\alpha}{k}}}{k \Gamma_{k}(\alpha)}, t \in[a, b]$ and $\varpi(s)=\frac{s^{\frac{\beta}{k}}}{k \Gamma_{k}(\beta)}, s \in[c, d], \alpha, \beta, k>0$ in Theorem 2, Theorem 3 and Theorem 4 , we have the following theorems.

Theorem 5. If $\sigma: \Delta \rightarrow \Re$ is of bounded variation on $\Delta$, then we have the following inequalities for $k$-Riemann-Liouville fractional integrals:

$$
\begin{gathered}
\mid \sigma(x, y)-\frac{\Gamma_{k}(\alpha+k)}{M(a, b ; x)}\left[\mathcal{I}_{a+}^{\alpha, k} \sigma(x, y)+\mathcal{I}_{b-}^{\alpha, k} \sigma(x, y)\right]- \\
\frac{\Gamma_{k}(\beta+k)}{N(c, d ; y)}\left[\mathcal{I}_{c+}^{\beta, k} \sigma(x, y)+\mathcal{I}_{d-}^{\beta, k} \sigma(x, y)\right]+\frac{\Gamma_{k}(\alpha+k) \Gamma_{k}(\beta+k)}{M(a, b ; x) N(c, d ; y)} \\
\left.\times{ }_{k} \mathcal{I}_{a+, c+}^{\alpha} \sigma(x, y)+{ }_{k} \mathcal{I}_{a+, d-}^{\alpha} \sigma(x, y)+{ }_{k} \mathcal{I}_{b-, c+}^{\alpha} \sigma(x, y)+{ }_{k} \mathcal{I}_{b-, d-}^{\alpha} \sigma(x, y)\right] \mid \\
\times\left[\int_{a}^{\alpha \beta} \int_{c}^{x}(x-t)^{\frac{\alpha}{k}-1}(y-s)^{\frac{\beta}{k}-1} \bigvee_{t}^{x} \bigvee_{s}^{y}(\sigma) d s d t\right. \\
+\int_{a}^{x} \int_{y}^{d}(x-t)^{\frac{\alpha}{k}-1}(s-y)^{\frac{\beta}{k}-1} \bigvee_{t}^{x} \bigvee_{y}^{s}(\sigma) d s d t
\end{gathered}
$$




$$
\begin{aligned}
& +\int_{x}^{b} \int_{c}^{y}(t-x)^{\frac{\alpha}{k}-1}(y-s)^{\frac{\beta}{k}-1} \bigvee_{x}^{t} \bigvee_{s}^{y}(\sigma) d s d t \\
& \left.+\int_{x}^{b} \int_{y}^{d}(t-x)^{\frac{\alpha}{k}-1}(s-y)^{\frac{\beta}{k}-1} \bigvee_{x}^{t} \bigvee_{y}^{s}(\sigma) d s d t\right] \leq \\
& \leq \frac{1}{M(a, b ; x) N(c, d ; y)} \times \\
& {\left[(x-a)^{\frac{\alpha}{k}}(y-c)^{\frac{\beta}{k}} \bigvee_{a}^{x} \bigvee_{c}^{y}(\sigma)+(x-a)^{\frac{\alpha}{k}}(d-y)^{\frac{\beta}{k}} \bigvee_{a}^{x} \bigvee_{y}^{d}(\sigma)+(b-x)^{\frac{\alpha}{k}}(y-c)^{\frac{\beta}{k}} \bigvee_{x}^{b} \bigvee_{c}^{y}(\sigma)\right.} \\
& \left.+(b-x)^{\frac{\alpha}{k}}(d-y)^{\frac{\beta}{k}} \bigvee_{x}^{b} \bigvee_{y}^{d}(\sigma)\right] \\
& \leq \frac{1}{M(a, b ; x) N(c, d ; y)}\left[\frac{1}{2}(b-a)+\left(x-\frac{a+b}{2}\right)\right]^{\frac{\alpha}{k}} \\
& {\left[\frac{1}{2}(b-c)+\left(y-\frac{c+d}{2}\right)\right]^{\frac{\beta}{k}} \bigvee_{a}^{b} \bigvee_{c}^{d}(\sigma)}
\end{aligned}
$$

for all $(x, y) \in \Delta$.

Theorem 6. If $\sigma: \Delta \rightarrow \Re$ is of bounded variation on $\Delta$, then we have the following inequalities for $k$-Riemann-Liouville fractional integrals:

$$
\begin{gathered}
\mid \sigma(x, y)-\frac{\Gamma_{k}(\alpha+k)}{M(a, b ; x)}\left[\mathcal{I}_{x+}^{\alpha, k} \sigma(b, y)+\mathcal{I}_{x-}^{\alpha, k} \sigma(a, y)\right]- \\
\frac{\Gamma_{k}(\beta+k)}{N(c, d ; y)}\left[\mathcal{I}_{y+k}^{\beta, k} \sigma(x, d)+\mathcal{I}_{y-}^{\beta, k} \sigma(x, c)\right]+\frac{\Gamma_{k}(\alpha+k) \Gamma_{k}(\beta+k)}{M(a, b ; x) N(c, d ; y)} \\
\times\left[{ }_{k} \mathcal{I}_{x+, y+}^{\alpha} \sigma(b, d)+{ }_{k} \mathcal{I}_{x+, y-}^{\alpha} \sigma(b, c)+{ }_{k} \mathcal{I}_{x-, y+}^{\alpha} \sigma(a, d)+{ }_{k} \mathcal{I}_{x-, y-}^{\alpha} \sigma(a, c)\right] \mid \\
\leq \frac{\alpha \beta}{k^{2} M(a, b ; x) N(c, d ; y)} \\
{\left[\int_{x}^{b} \int_{y}^{d}(b-t)^{\frac{\alpha}{k}-1}(d-s)^{\frac{\beta}{k}-1} \bigvee_{x} \bigvee_{y}(\sigma) d s d t+\int_{x}^{b} \int_{c}^{y}(b-t)^{\frac{\alpha}{k}-1}(s-c)^{\frac{\beta}{k}-1} \bigvee_{x}^{t} \bigvee_{s}^{y}(\sigma) d s d t\right.} \\
\left.+\int_{a}^{x} \int_{y}^{d}(t-a)^{\frac{\alpha}{k}-1}(d-s)^{\frac{\beta}{k}-1} \bigvee_{t}^{x} \bigvee_{y}(\sigma) d s d t+\int_{a}^{y}(t-a)^{\frac{\alpha}{k}-1}(s-c)^{\frac{\beta}{k}-1} \bigvee_{t}^{x} \bigvee_{s}^{y}(\sigma) d s d t\right]
\end{gathered}
$$




$$
\begin{gathered}
\leq \frac{1}{M(a, b ; x) N(c, d ; y)}\left[(b-x)^{\frac{\alpha}{k}}(d-y)^{\frac{\beta}{k}} \bigvee_{x}^{b} \bigvee_{y}^{d}(\sigma)+(b-x)^{\frac{\alpha}{k}}(y-c)^{\frac{\beta}{k}} \bigvee_{x}^{b} \bigvee_{c}^{y}(\sigma)\right. \\
\left.+(x-a)^{\frac{\alpha}{k}}(d-y)^{\frac{\beta}{k}} \bigvee_{a}^{x} \bigvee_{c}^{y}(\sigma)+(x-a)^{\frac{\alpha}{k}}(y-c)^{\frac{\beta}{k}} \bigvee_{a}^{x} \bigvee_{c}^{y}(\sigma)\right] \leq \\
\leq \frac{1}{M(a, b ; x) N(c, d ; y)}\left[\frac{1}{2}(b-a)+\left(x-\frac{a+b}{2}\right)\right]^{\frac{\alpha}{k}} \\
\cdot\left[\frac{1}{2}(b-c)+\left(y-\frac{c+d}{2}\right)\right]^{\frac{\beta}{k}} \bigvee_{a}^{b} \bigvee_{c}^{d}(\sigma)
\end{gathered}
$$

for all $(x, y) \in \Delta$.

Theorem 7. If $\sigma: \Delta \rightarrow \Re$ is of bounded variation on $\Delta$, then we have the following inequalities for $k$-Riemann-Liouville fractional integrals:

$$
\begin{aligned}
& \mid \frac{\sigma(a, c)+\sigma(a, d)+\sigma(b, c)+\sigma(b, d)}{4} \frac{(b-a)^{\frac{\alpha}{k}}(d-c)^{\frac{\beta}{k}}}{\Gamma_{k}(\alpha+k) \Gamma_{k}(\beta+k)} \\
& -\frac{(b-a)^{\frac{\alpha}{k}}}{4 \Gamma_{k}(\alpha+k)}\left[\mathcal{I}_{c+}^{\beta, k} \sigma(b, d)+\mathcal{I}_{d-}^{\beta, k} \sigma(b, c)+\mathcal{I}_{c+}^{\beta, k} \sigma(a, d)+\mathcal{I}_{d-}^{\beta, k} \sigma(a, c)\right] \\
& -\frac{(d-c)^{\frac{\beta}{k}}}{4 \Gamma_{k}(\beta+k)}\left[\mathcal{I}_{a+}^{\alpha, k} \sigma(b, d)+\mathcal{I}_{b-}^{\alpha, k} \sigma(a, d)+\mathcal{I}_{a+}^{\alpha, k} \sigma(b, c)+\mathcal{I}_{b-}^{\alpha, k} \sigma(a, c)\right] \\
& +\frac{1}{4}\left[{ }_{k} \mathcal{I}_{a+, c+}^{\alpha} \sigma(b, d)+{ }_{k} \mathcal{I}_{a+, d-}^{\alpha} \sigma(b, c)+{ }_{k} \mathcal{I}_{b-, c+}^{\alpha} \sigma(a, d)+{ }_{k} \mathcal{I}_{b-, d-}^{\alpha} \sigma(a, c)\right] \mid \\
& \leq \frac{1}{4 k^{2} \Gamma_{k}(\alpha) \Gamma_{k}(\beta)} \times \\
& {\left[\int_{a}^{b} \int_{c}^{d}(b-t)^{\frac{\alpha}{k}-1}(d-s)^{\frac{\beta}{k}-1} \bigvee_{t}^{b} \bigvee_{s}^{d}(\sigma) d s d t+\int_{a}^{b} \int_{c}^{d}(b-t)^{\frac{\alpha}{k}-1}(s-c)^{\frac{\beta}{k}-1} \bigvee_{t}^{b} \bigvee_{c}^{s}(\sigma) d s d t+\right.} \\
& \left.\int_{a}^{b} \int_{c}^{d}(t-a)^{\frac{\alpha}{k}-1}(d-s)^{\frac{\beta}{k}-1} \bigvee_{a}^{t} \bigvee_{s}^{d}(\sigma) d s d t+\int_{a}^{b} \int_{c}^{d}(t-a)^{\frac{\alpha}{k}-1}(s-c)^{\frac{\beta}{k}-1} \bigvee_{a}^{t} \bigvee_{c}^{s}(\sigma) d s d t\right] \\
& \leq \frac{(b-a)^{\frac{\alpha}{k}}(d-c)^{\frac{\beta}{k}}}{\Gamma_{k}(\alpha+k) \Gamma_{k}(\beta+k)} \bigvee_{a}^{b} \bigvee_{c}^{d}(\sigma)
\end{aligned}
$$

for all $(x, y) \in \Delta$. 


\section{Conclusion}

In this paper, we established some identities for generalized fractional integrals. Utilizing these identities, some Ostrowski and Midpoint type inequalities for generalized fractional integrals for functions of bounded variation with two variables are obtained. Moreover, some new inequalities involving $k$-Riemann-Liouville fractional integrals are presented as special cases of our main results. Since functions with bounded variation with two variables have large applications in many mathematical areas, our results can be applied to obtain several new fascinating inequalities in convex analysis, special functions, quantum mechanics, related optimization theory, mathematical inequalities and also may stimulate further research in different areas of pure and applied sciences.

Conflict of interest. All the authors declares that they have no conflict of interest with anyone.

\section{REFERENCES}

[1] A. Akkurt, M. Z. Sarikaya, H. Budak, H. Yildirim, On the Hadamard's type inequalities for co-ordinated convex functions via fractional integrals, Journal of King Saud UniversityScience, 29, (2017), 380-387.

[2] H. Budak, M. Z. Sarikaya, A companion of Ostrowski type inequalities for mappings of bounded variation and some applications, Trans. A. Razmadze Math. Inst., 171, (2017), $136-143$.

[3] H. Budak, M. Z. Sarikaya, A. Qayyum, Improvement in companion of Ostrowski type inequalities for mappings whose first derivatives are of bounded variation and application, Filomat, 31(16), (2017), 5305-5314.

[4] H. Budak, M. Z. Sarikaya, A new generalization of Ostrowski type inequalities for mappings of bounded variation, Lobachevskii J. Math., 39(9), (2018), 1320-1326.

[5] H. Budak, M. Z. Sarikaya, On generalization Ostrowski type inequalities for functions of two variables with bounded variation and applications, Palest. J. Math., 5(1), (2016), 86-97.

[6] H. Budak, M. Z. Sarikaya, On Ostrowski type inequalities for functions of two variables with bounded variation, Int. J. Anal. Appl., 12(2), (2016), 142-156.

[7] H. Budak, M. Z. Sarikaya, A companion of generalization of Ostrowski type inequalities for functions of two variables with bounded variation, Appl. Comput. Math., 15(3), (2016), $297-312$.

[8] H. Budak, P. Agarwal, On Hermite-Hadamard type inequalities for co-ordinated convex mappings utilizing generalized fractional integrals, Accepted as an Book Chaper, Fractional Differentiation and its Applications, Springer.

[9] P. Cerone, S. S. Dragomir, C. E. M. Pearce, A generalized trapezoid inequality for functions of bounded variation, Turk. J. Math., 24, (2000), 147-163.

[10] J. A. Clarkson, C. R. Adams, On definitions of bounded variation for functions of two variables, Bull. Amer. Math. Soc., 35, (1933), 824-854.

[11] S. S. Dragomir, Ostrowski type inequalities for Riemann-Liouville fractional integrals of bounded variation, Holder and Lipschitzian functions, RGMIA Research Report Collection, 20, (2017), Article 48.

[12] S. S. Dragomir, Ostrowski and Trapezoid type inequalities for Riemann-Liouville fractional integrals of functions with bounded variation, RGMIA Research Report Collection, 20, (2017), Article 52.

[13] S. S. Dragomir, Ostrowski type inequalities for generalized Riemann-Liouville fractional integrals of functions with bounded variation, RGMIA Research Report Collection, 20, (2017), Article 58. 
[14] S. S. Dragomir, On the midpoint quadrature formula for mappings with bounded variation and applications, Kragujevac J. Math., 22, (2000), 13-19.

[15] S. S. Dragomir, On the Ostrowski's integral inequality for mappings with bounded variation and applications, Math. Inequal. Appl., 4(1), (2001), 59-66.

[16] S. Erden, Some perturbed inequalities of Ostrowski type for funtions whose nth derivatives are of bounded, Iran. J. Math. Sci. Inform., In press, 2019.

[17] S. Erden, H. Budak, M. Z. Sarikaya, Fractional Ostrowski type inequalities for functions of bounded variation with two variables, Submitted, 2019 https://www.researchgate.net/publication/336698773.

[18] G. Farid, Some new Ostrowski type inequalities via fractional integrals, Int. J. Anal. Appl., 14(1), (2017), 64-68.

[19] G. Farid, A. Rehman, M. Zahra, On Hadamard inequalities for $k$-fractional integrals, Nonlinear Funct. Anal. Appl., 21(3), (2016), 463-478.

[20] R. Gorenflo, F. Mainardi, Fractional calculus: integral and differential equations of fractional order, Springer Verlag, Wien (1997), 223-276.

[21] M. Jleli, B. Samet, On Hermite-Hadamard type inequalities via fractional integrals of a function with respect to another function, J. Nonlinear Sci. Appl., 9, (2016), 1252-1260.

[22] A. A. Kilbas, H. M. Srivastava, J. J. Trujillo, Theory and Applications of Fractional Differential Equations, North-Holland Mathematics Studies, 204, Elsevier Sci. B.V., Amsterdam, 2006.

[23] M. A. Latif, S. Hussain, New inequalities of Ostrowski type for co-ordinated convex functions via fractional integrals, J. Fract. Calc. Appl., 2(9), (2012), 1-15.

[24] S. Miller, B. Ross, An introduction to the Fractional Calculus and Fractional Differential Equations, John Wiley \& Sons, USA, 1993.

[25] S. Mubeen, G. M. Habibullah, $k$-Fractional integrals and application, Int. J. Contemp. Math. Sciences, 7(2), (2012), 89-94.

[26] A. M. Ostrowski, Über die absolutabweichung einer differentiebaren funktion von ihrem integralmitelwert, Comment. Math. Helv., 10, (1938), 226-227.

[27] I. Podlubni, Fractional Differential Equations, Academic Press, San Diego, 1999.

[28] M. Z. Sarikaya, H. Budak, Generalized Hermite-Hadamard type integral inequalities for fractional integrals, Filomat, 30(5), (2016), 1315-1326.

[29] M. Z. Sarikaya, F. Ertuğral, On the generalized Hermite-Hadamard inequalities, Annals of the University of Craiova-Mathematics and Computer Science Series, In Press.

[30] M. Z. Sarikaya, E. Set, H. Yaldiz, N. Basak, Hermite-Hadamard's inequalities for fractional integrals and related fractional inequalities, Math. Comput. Modelling, 57, (2013), 24032407.

[31] M. Z. Sarikaya, On the Hermite-Hadamard-type inequalities for co-ordinated convex function via fractional integrals, Integral Transforms Spec. Funct., 25(2), (2014), 134-147.

[32] M. Z. Sarikaya, H. Filiz, Note on the Ostrowski type inequalities for fractional integrals, Vietnam J. Math., 42(2), (2014), 187-190.

[33] E. Set, New inequalities of Ostrowski type for mappings whose derivatives are s-convex in the second sense via fractional integrals, Comput. Math. Appl., 63(7), (2012), 1147-1154.

[34] H. Yaldiz, M. Z. Sarikaya, Z. Dahmani, On the Hermite-Hadamard-Fejér-type inequalities for co-ordinated convex functions via fractional integrals, Int. J. Optim. Control, Theor. Appl. (IJOCTA), 7(2), (2017), 205-215.

[35] M. E. Yildirim, M. Z. Sarikaya, H. Budak, H. Yildirim, Some Hermite-Hadamard type inequalities for coordinated-convex functions via generalized fractional integrals, (2017), https://www.researchgate.net/publication/321803898.

[36] P. O. Mohammed, T. Abdeljawad, S. Zeng, A. Kashuri, Fractional Hermite-Hadamard integral inequalities for a new class of convex functions, Symmetry, 12, (2020), 1485.

[37] A. Kashuri, R. Liko, Some new Hermite-Hadamard type inequalities and their applications, Stud. Sci. Math. Hung., 56(1), (2019), 103-142. 
[38] A. Kashuri, S. Iqbal, S. I. Butt, J. Nasir, K. S. Nisar, T. Abdeljawad, Trapezium-type inequalities for $k$-fractional integral via new exponential-type convexity and their applications, J. Math., 2020, (2020), Article ID 8672710, 12 pp.

[39] A. Kashuri, B. Meftah, P. O. Mohammed, Some weighted Simpson type inequalities for differentiable s-convex functions and their applications, J Frac Calc \& Nonlinear Sys, 1(1), (2021), 75-94.

[40] D. Baleanu, A. Kashuri, P. O. Mohammed, B. Meftah, General Raina fractional integral inequalities on coordinates of convex functions, Adv. Difference Equ., 2021(82), (2021), 23 $\mathrm{pp}$

1 Department of Mathematics, Faculty of Technical Science,

University "Ismail Qemali", 9400, Vlora, Albania

Email address: artionkashuri@gmail.com

Email address: rozanaliko86@gmail.com

2 Department of Mathematics, Faculty of Science and Arts, Düzce University, Düzce, Turkey

Email address: hsyn.budak@gmail.com

Email address: kubilayozcelik@windowslive.com

${ }^{3}$ School of Mathematical Sciences,

Nanjing Normal University, Nanjing, 210023, China

Email address: mahr.muhammad.aamir@gmail.com

Received: 10.2 .2021

Revised: $\quad 7.5 .2021$

Accepted: 29.5.2021 